which takes a minimum of 46 hours. The implications of this rapid fixing/decalcifying protocol are that paraffin wax sections of a trephine biopsy specimen can be ready to examine the day after the biopsy specimen is taken. In practice, bone marrow trephine biopsy specimens can be processed after as little as three hours in isotonic TCA, with good results. However, to be certain of adequate fixation we stipulate a minimum fixation time of five hours.

Morphological preservation at 80 hours was better using isotonic TCA. Trichloroacetic acid is known to increase tissue volume, but the addition of $\mathrm{NaCl}$ to form an isotonic solution seems to reduce this effect. If there is a risk of excessive exposure to TCA, such as may occur if a biopsy specimen is left in TCA from Friday over the weekend to Monday, it is advisable to use an isotonic solution to prevent unwanted morphological deterioration.

Processing bone marrow trephine biopsy specimens in isotonic TCA not only cuts down on conventional processing time but also results in superior morphological preservation than that achievable using formalin fixation.
Shrinking artefact is reduced and nuclear detail enhanced thus more closely approximating the standard of plastic sections.

1 Bartl R, Frisch B, Burkhardt R. Introduction and structure and function of the normal bone marrow. In: Bone marrow biopsies revisited. $A$ new dimension for haematologic malignancies. 2nd edn. Basel: Karger, 1985:1-3, 9-15.

2 Holdrinet R, von Egmund J, Wessels J, Haanen C. A method for quantification of peripheral blood admixtures in bone marrow aspirates. Exp Haematol 1980;8:103-7.

3 Van der Valk P, Mullink H, Huijgens PC, Tadema TM, Vos W, Meijer CJLM. Immunohistochemistry in bone marrow diagnosis. Value of a panel of monoclonal antibodies on routinely processed bone marrow biopsies. Am $\mathcal{J}$ Surg Pathol 1989;13:97-106.

4 Wilkins BS, O'Brien CJ. Techniques for obtaining differential cell counts from bone marrow trephine biopsy specimens. f Clin Pathol 1988;41:558-61.

5 Islam A, Frisch B. Plastic embedding in routine histology I: Preparation of semithin sections of undecalcified marrow cores. Histopathology 1985;9:1263-74.

6 Anonymous. Plastic or paraffin? [Editorial.] Lancet 1989 ;i:139-40.

7 Bancroft JD, Stevens A. Frozen and related sections. In: Theory and practice of histological techniques. 2nd edn. Edinburgh: Churchill Livingstone, 1982:82-90.

8 Hall PA, Stearn PM, Butler MG, D'Ardenne AJD. Acetone/ periodate-lysine-paraformaldehyde (PLP) fixation and perioded mor improved morphology of cryostat sections for imm un

Kiernan JA. Histological and histochemical methods: In: Theory and practice. Oxford: Pergamon Press, 1981:

\title{
Two techniques for electron opaque staining of elastic fibres using tannic acid in fresh and formalin fixed tissue
}

\author{
A Haidar, T A Ryder, M A Mobberley, J S Wigglesworth
}

\author{
Department of \\ Histopathology, Royal \\ Postgraduate Medical \\ School, Hammersmith \\ Hospital, Ducane \\ Road, London \\ W12 0HS \\ A Haidar \\ J S Wigglesworth \\ Electron microscopy \\ unit, Queen \\ Charlotte's and \\ Chelsea Hospital, \\ London \\ M A Mobberley \\ T A Ryder \\ Correspondence to: \\ Professor J S Wigglesworth \\ Accepted for publication \\ 29 November 1991
}

\begin{abstract}
Two electron microscopic staining techniques, one using tannic acid-glutaraldehyde as a fixative, and the other using tannic acid-uranyl acetate solution as a stain on ultra-thin sections of glutaraldehyde fixed material, were directly compared for elastic fibre staining on several human and animal tissues. Various concentrations of tannic acid were compared using both techniques. The two techniques were also compared on formalin fixed tissues.

The use of tannic acid-uranyl acetate solution as a stain on processed tissue is by far the more consistent technique and achieves equally good results on glutaraldehyde or formalin fixed tissue.

It is suggested that the use of the term tannic acid technique/method should be reserved for this particular method to achieve a meaningful interpretation of results in scientific papers.
\end{abstract}

Elastic fibres are components of the extracellular matrix and are present in most organs.
They are synthesised by connective tissue cells. ${ }^{1}$ Morphogenesis of normal and abnormal elastic fibres in adult and human fetal lung has recently been described using two different electron microscopic techniques ${ }^{23}$ involving the use of tannic acid, along with other methods. ${ }^{4-6}$ The exact mechanism involved in the interaction between tannic acid and elastin is unknown, although Hayat has pointed out some possible mechanisms. ${ }^{7}$ These include ionic interactions between carboxylate anions from tannic acid and cationic sites on the protein. Alternatively, hydrogen bonding may be involved. At an alkaline $\mathrm{pH}$ there may be binding of anionic oxygen to a cationic group such as ammonium in elastin. ${ }^{8}$ Kageyama and colleagues, ${ }^{9}$ using a modification of the original Kajikawa technique, ${ }^{2}$ have in fact shown that the maximum tannic acid staining of elastic fibres is in the $\mathrm{pH}$ range $7 \cdot 0-9 \cdot 0$.

The claim that tannic acid enhances staining of connective tissue elements including elastic fibres ${ }^{10}$ led us to compare these two tannic acid techniques $^{23}$ to see whether they could be used interchangeably. The two methods were applied to both formalin and glutaraldehyde fixed tissues. 


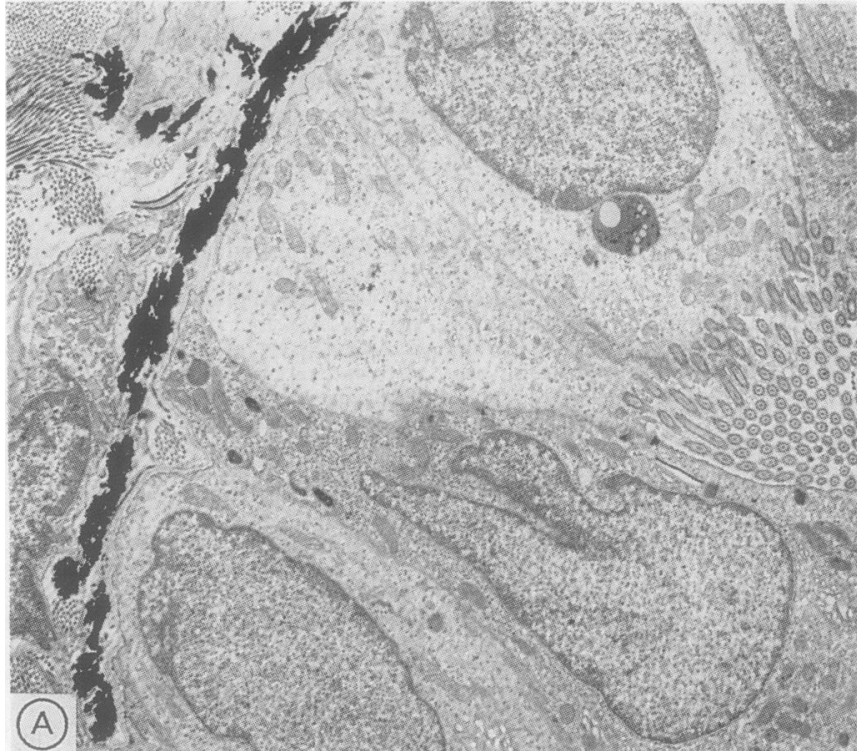

Figure $1 A$ Electron micrograph showing dense staining of the elastin in the wall of the rat bronchus (tannic acid-uranyl acetate stain).

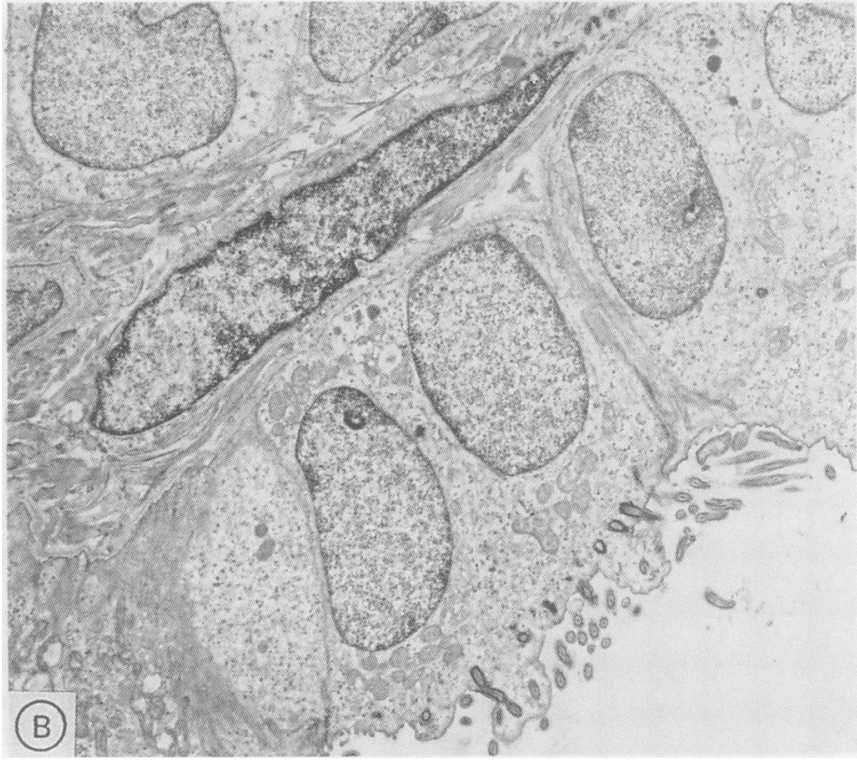

Figure $1 B \quad$ No staining of the elastin in a comparable area in the wall of the rat bronchus (tannic acid-glutaraldehyde fixation technique).

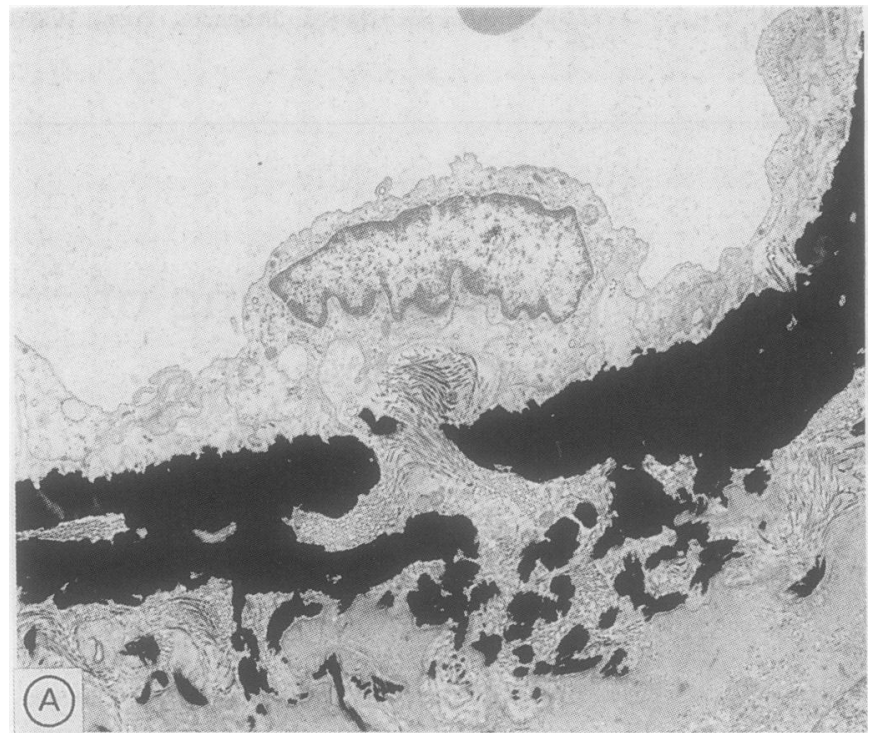

Figure $2 A$ Dense staining of the abundant elastin in the wall of the rat aorta (tannic acid-uranyl acetate stain).

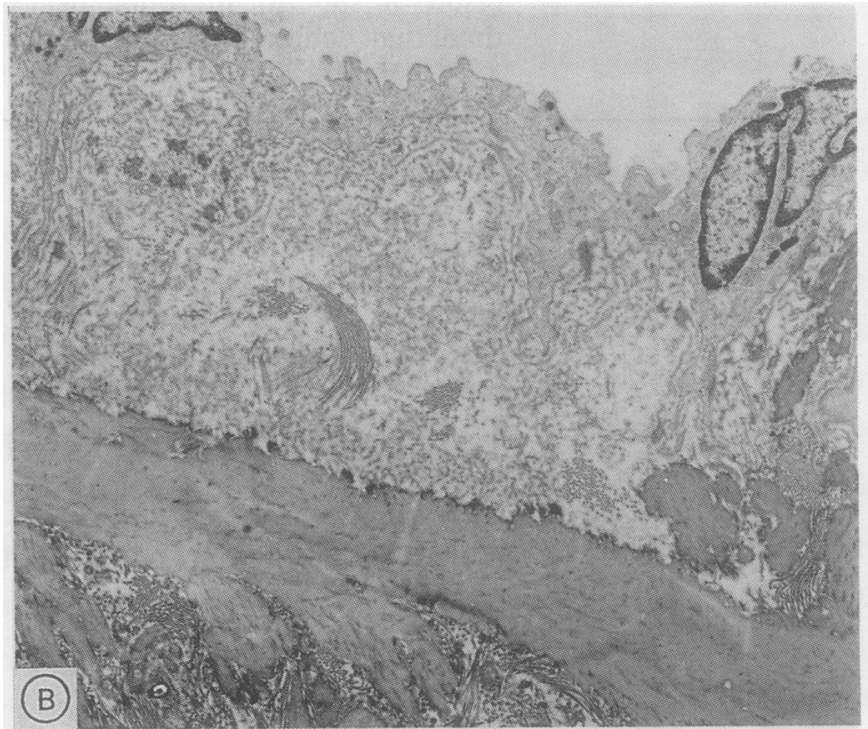

Figure 2B No demonstrable staining of the elastin in the wall of the rat aorta with tannic acid-glutaraldehyde fixation technique.

\section{Methods}

Rat aorta and lung blocks and human fetal lung and kidney blocks were fixed in 3\% glutaraldehyde in $0 \cdot 1 \mathrm{M}$ cacodylate buffer $(\mathrm{pH}$ $7 \cdot 2$ ) with the addition of $1 \%, 0.5 \%$, or $0.25 \%$ tannic acid. Comparable blocks from these tissues were fixed in $3 \%$ buffered glutaraldehyde alone. These were post-fixed in $1 \%$ osmium tetroxide, dehydrated, and embedded in Araldite. Ultra-thin sections from glutaraldehyde fixed tissue were mounted on copper grids and stained with freshly made tannic acid-uranyl acetate solution, prepared as described previously. ${ }^{2}$ The sections were stained by floating them on single drops of this solution for 10 minutes, and after a thorough rinse in distilled water they were dried and stained with lead citrate for five minutes. The ultra-thin sections from tannic acid-glutaraldehyde fixed tissue were routinely stained for one hour in saturated alcoholic uranyl acetate followed by five minutes in lead citrate.

In the second part of this investigation $10 \%$ neutral buffered formalin was substituted for glutaraldehyde for comparison of the two techniques.

\section{Results}

Use of tannic acid-uranyl acetate on routinely fixed tissue gave precise and highly specific localisation of elastin aggregates and elastic fibres in all the tissues examined. Figures 1A and $2 \mathrm{~A}$ show elastic fibres in the rat bronchus and the wall of the aorta respectively. The results were consistent with excellent reproducibility, and background non-specificity was not a problem. Substitution of $10 \%$ neutral buffered formalin for glutaraldehyde did not alter the outcome. Figure 3 shows clearly delineated inner elastic lamina in the vessel wall of a formalin fixed human fetal lung. 


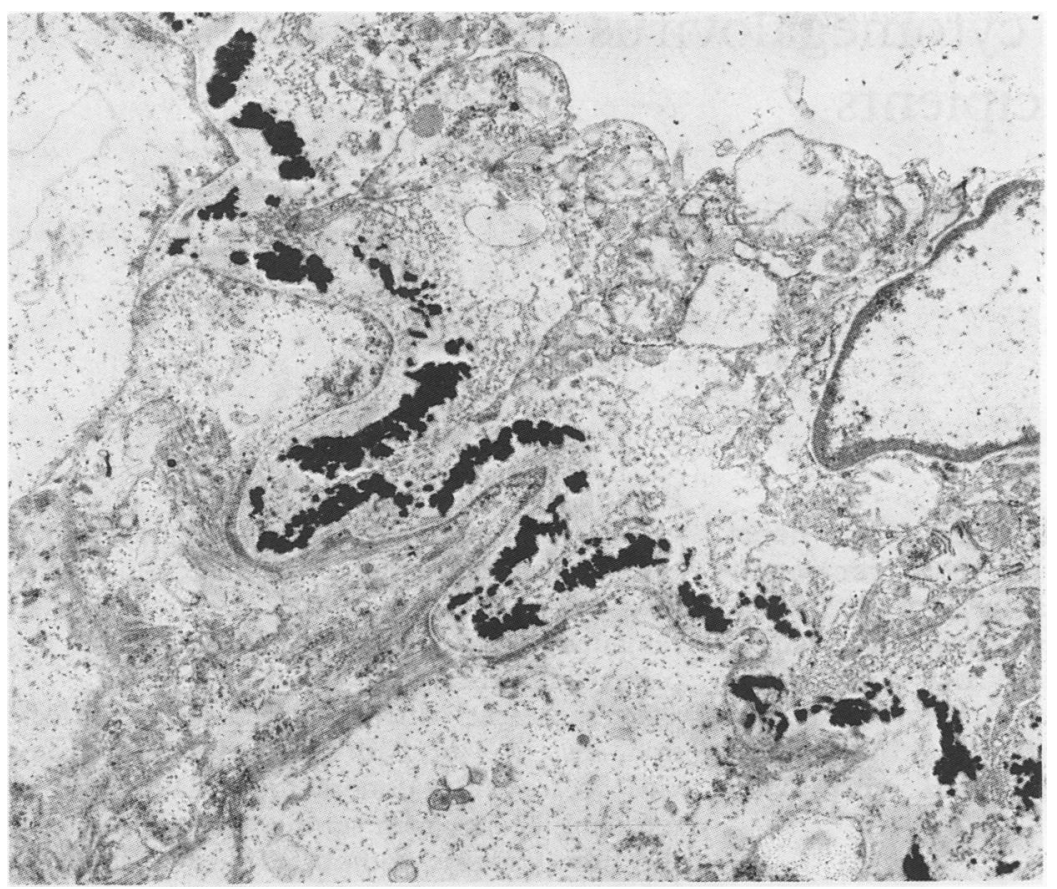

Figure 3 Clearly delineated dense staining of the internal elastic lamina in the vessel wall of a human fetal lung fixed in formalin (tannic acid-uranyl acetate stain).

By comparison, results obtained by using the tannic acid-glutaraldehyde fixation technique showed poor (almost non-existent) localisation at comparable sites in the rat bronchus (fig 1B) and the wall of the rat aorta (fig 2B).

\section{Discussion}

The term tannic acid technique/method has been used interchangeably ${ }^{45}$ for these two essentially different methods. In view of the findings in our present study this practice could be a cause of much confusion especially when dealing with recently formed elastin during fetal lung development or abnormal morphogenesis of elastic fibres in diseases such as pulmonary emphysema.
Our main criticisms of the tannic acidglutaraldehyde fixation technique are that (i) the results are poor and inconsistent; and (ii) the non-specificity of the method can cause elastin apparently to localise in some areas and not in others, thereby facilitating mistakes. Therefore, we do not recommend its use as a technique for visualising elastic fibres.

We also found that substituting neutral buffered formalin for glutaraldehyde did not alter the result with either method. This suggests that routinely formalin fixed tissue can potentially be used for electron microscopic observation of elastin development in fetal organs and also for studying elastosis in soft tissue pathology such as granular cell tumours.

This study was partly funded by an ORS award to Dr Haidar.

1 Serafini-Fracassini A. Elastogenesis in embryonic and post natal development. In: Ruggeri A, Motta PM, eds. Ultrastructure of the connective tissue matrix. Boston: Martinus Nijhoff, 1984:140-50.

2 Kajikawa K, Yamaguchi T, Katsuda S, Miwa A. An improved electron stain for elastic fibres using tannic acid. $\mathcal{F}$ Electron Microsc 1975;24:287-9.

3 Futaesaku Y, Mizuhira V, Nakamura H. A new fixation method using tannic acid for electron microscopy and some observations of biological specimens. Proceeding of the Fourth International Congress in Histochemistry. Kyoto: Society of Histochemistry and Cytochemistry, 1972:155-6.

4 Fukuda Y, Masuda Y, Ishizaki M, et al. Morphogenesis of abnormal elastic fibres in lungs of patients with panacinar and centriacinar emphysema. Hum Pathol 1989;20: and-9.

5 Nakamura Y, Fukuda S, Hashimoto T, et al. Pulmonary elastic fibres in normal human lung development and in pathological conditions. Pediatr Pathol 1990;10:689-706.

6 Haidar A, Ryder TA, Wigglesworth JS. An electron microscopic demonstration of absent elastin development in hypoplastic lungs associated with oligohydramnios. Histopathology 1991;18:471-3.

7 Hayat MA. Principles and techniques of electron microscopybiological applications. 3rd edn. London: Macmillan, 1989:298-9.

8 Anwar RA, Oda G. The biosynthesis of desmosine and isodesmosine. F Biol Chem 1966;241:4638-41.

9 Kageyama M, Takagi M, Parmley RT, Toda M, Hirayama H, Toda Y. Ultrastructural visualization of elastic fibres with a tannate-metal salt method. Histochem with a tannate-

10 Cotta-Pereira G, Guerra Rodrigo F, David-Ferreira JF. The use of tannic acid-glutaraldehyde in the study of elastic and elastic-related fibres. Stain Technol 1976;51:7-11. 